\title{
Review of recent optical issues in LEP
}

\author{
F. Ruggiero, \\ CERN \\ CH-1211 Geneva 23
}

\section{Abstract}

We review some optical measurements and correction strategies adopted for the new lattice with $90^{\circ}$ phase advance used in LEP during 1992. In particular, we compare three different techniques used to measure beta-beating: a multi-turn orbit measurement in presence of betatron excitation, a method based on the variation of chromaticity due to opposite trims in the strength of two sextupole families and an orbit measurement with two orthogonal kicks. The average vertical beating measured by these three methods (up to $37 \%$, depending on the optical configuration) shows a substantial agreement among them. We also discuss a resonant method of correction for residual dispersion by special orbit bumps. The amplification factors for such bumps range from 200 to more than 700 , i.e., a $1 \mathrm{~mm}$ orbit bump can give rise to more than $70 \mathrm{~cm}$ peak dispersion and these bumps have been routinely used to control beam size and optimize machine performance without any appreciable effect on the closed orbit.

\section{Measurements of Beta-Beating}

During 1992, the behaviour of the beta-functions in LEP did not correspond to the theoretical predictions. In particular, for an optical configuration supposed to yield a vertical beta value of $5 \mathrm{~cm}$ at the IP's, the actual measured beta value was around $7 \mathrm{~cm}$. This effect was corrected by applying an empirical trim $\Delta K / K=7.24 \times 10^{-4}$ to the strength of the low-beta quadrupoles (QS0's). A more general study of the associated beta-beating was then started to understand the origin of such discrepancies and their consequences on the available aperture of the machine. The three different techniques used to measure the beating of the beta-functions [1] are described below.

\section{A. Multi-turn orbit measurement}

This method consists in the analysis of the turn by turn readings of the monitors (up to 1024 turns) in presence of betatron excitation. The data is then Fourier analyzed at the excitation frequency (usually close to the betatron frequency) yielding the amplitude and phase of the driven betatron oscillations around the machine [2].

\section{B. Sextupole method}

The strength of one of the two defocussing sextupole families (SDI) is increased by some amount, whereas the strength of the second family (SD2) is decreased by the same amount. This should leave the vertical chromaticity
$Q^{\prime}$ of the machine unchanged if the $\beta$-values are the same in all the SD sextupoles, since the phase advance between two successive SD2 is $\pi$ (this still holds in case of beating of the dispersion). The variation of chromaticity is related to the difference between the vertical $\beta$ 's at the SD1 and $\mathrm{SD} 2$ sextupoles by [3]:

$$
\Delta Q^{\prime}=\frac{1}{4 \pi} D_{x, S D} L_{S D} \Delta K_{S D 1}^{\prime}\left(\beta_{S D 2}-\beta_{S D 1}\right) .
$$

\section{Orthogonal kicks}

Closed orbit distortions are created by subsequently exciting two corrector magnets with a phase advance of $\pi / 2$ between them. In an ideal machine without beating, squaring the readings of the two orbits and adding them eliminates the phase-dependent term in the orbit response, and directly yields the values of the $\beta$-function. In presence of beating, however, the values $\beta_{1}$ and $\beta_{2}$ at the two correctors may be different and the phase advance between them may deviate from the nominal value $\pi / 2$ by some amount $\epsilon$. In general, the $\beta$-function is given by

$$
\beta(s)=a y_{1}^{2}(s)+b y_{2}^{2}(s)+c y_{1}(s) y_{2}(s),
$$

where $y_{1}(s)$ and $y_{2}(s)$ denote the measured (difference) orbits corresponding to the excitation of each corrector and the three coefficients $a, b$ and $c$ have the following theoretical values:

$$
a=F / \beta_{1}, \quad b=F / \beta_{2}, \quad c=-2 F \sin (\epsilon) / \sqrt{\beta_{1} \beta_{2}} .
$$

Here $F=\left[2 \sin (\pi Q) / \cos (\epsilon) \Delta y^{\prime}\right]^{2}$ and $\Delta y^{\prime}$ is the common (angular) strength of the two correctors.

The coefficients $a, b$ and $c$ have been estimated using two independent methods, both giving the correct result when applied to orbits simulated with MAD. The first method makes use of the (vertical) orbit values $y_{1}\left(s_{1}\right), y_{2}\left(s_{2}\right)$ at the PU's closest to the correctors in order to obtain $\beta_{1}$ and $\beta_{2}$. It can be shown that the 'cross terms' $y_{1}\left(s_{2}\right)$ and $y_{2}\left(s_{1}\right)$ should have the same value $y_{12}$ given by

$$
y_{12}=\frac{1}{2} \Delta y^{\prime} \sqrt{\beta_{1} \beta_{2}}\left[\frac{\sin (\epsilon)}{\tan (\pi Q)}+\cos (\epsilon)\right] \text {. }
$$

Therefore the equality of the cross terms can be used as a self-consistency test and $\epsilon$ can be estimated from their common value $y_{12}$. This method is independent of the nominal $\beta$-function, but requires the measured tune $Q$.

A second method to estimate the coefficients $a, b$ and $c$ consists in a five-parameter fit of the nominal $\beta$-function $\beta_{N}=a y_{1}^{2}+b y_{2}^{2}+c y_{1} y_{3}-\beta_{N}\left[A \cos \left(2 \phi_{N}\right)+B \sin \left(2 \phi_{N}\right)\right]$, 


\begin{tabular}{||c|c|c||c|c||c|c||}
\hline \multirow{2}{*}{} & \multicolumn{2}{|c||}{$\beta_{y}^{*}=21 \mathrm{~cm}$} & \multicolumn{2}{c||}{$\beta_{y}^{*}=7 \mathrm{~cm}$} & \multicolumn{2}{c||}{$\beta_{y}^{*}=5 \mathrm{~cm}$} \\
Octant & multi-turn & kicks & multi-turn & kicks & multi-turn & kicks \\
\hline 1 & 19 & 17 & 41 & 41 & 16 & 19 \\
2 & 14 & 15 & 42 & 40 & 18 & 24 \\
3 & 9 & 6 & 33 & 29 & 6 & 12 \\
4 & 11 & 5 & 34 & 29 & 7 & 11 \\
5 & 10 & 4 & 34 & 28 & 12 & 9 \\
6 & 7 & 9 & 32 & 33 & 9 & 14 \\
7 & 16 & 4 & 42 & 30 & 24 & 10 \\
8 & 13 & 6 & 40 & 36 & 18 & 14 \\
\hline$(\Delta \beta / \beta)$ & 12 & 8 & 37 & 33 & 14 & 14 \\
\hline sextupoles & \multicolumn{2}{|c|}{9} & & \multicolumn{2}{|c|}{37} & \multicolumn{2}{|c||}{13} \\
\hline
\end{tabular}

Table 1: Vertical beta-beating (in per cent) for the detuned optics, for the nominal squeezed optics and for the squeezed optics with trimmed QSO's: results for multi-turn analysis and orthogonal kicks are octant by octant, while only the average beating is given for the sextupole method.

where the harmonic terms in square bracket take into account the beating at twice the nominal betatron phase $\phi_{N}$. Applying this method to simulated orbits, we have found that the correlation of the fit becomes poor when the betabeating is produced by a few localized sources (e.g. QSO's), but that a good correlation can be recovered by introducing a different harmonic amplitude for each arc: therefore we effectively perform a fit with 3 plus 8 parameters. This method is more stable against $\mathrm{PU}$ noise, since it makes use of the information at all the PU's, but the resulting amplitude of beta-beating in each arc depends somewhat on the criterion adopted for the rejection of bad PU's.

We have used the first method to have a rough estimate of $a, b$ and $c$ : then we have discarded monitors where $\Delta \beta / \beta$ was larger then 3 times its r.m.s. value and finally we have used the second method, based on the fit, to arrive at our final result. Typical values for $a, b$ and $c$ were $1.5,1$ and 0.25 , respectively, thus showing a significant deviation from the simple rule of summing the squares of the two orbits.

\section{Comparison of the results}

In Table 1, we report the vertical beta-beating measured by the multi-turn and by the orthogonal kick method in each LEP octant for three different optics, together with the corresponding average beating measured by the sextupole method. In Table 2, which refers to a perturbed squeezed optics, the results of the sextupole method are reported quadrant by quadrant. The average vertical betabeating measured by our three independent methods shows a substantial agreement among them, with the results of the sextupole method typically lying below those of the multi-turn and above those of the orthogonal kicks. The comparison of the beating amplitudes octant by octant suggests a larger spread in the results of the three methods, possibly associated with the criterion adopted for bad PU rejection. Finally, all methods confirm a large vertical beating for the nominal machine with $\beta_{y}^{*}=7 \mathrm{~cm}$ and a significant reduction of this beating as a consequence of the trim applied to the QSO magnets in order to bring $\beta_{y}^{*}$ down to $5 \mathrm{~cm}$.

During the last LEP shutdown, the longitudinal position of the QSO and QS1 magnets was found to be wrong by significant amounts (up to $9 \mathrm{~mm}$ ). According to recent simulations [4], these quadrupole shifts are largely suffcient to explain the observed beta-beating.

\begin{tabular}{||c||c|c|c||}
\hline Octant & multi-turn & kicks & sextupoles \\
\hline 1 & 22 & 16 & 22 \\
2 & 22 & 18 & \\
3 & 20 & 10 & 15 \\
4 & 20 & 12 & \\
5 & 21 & 14 & 23 \\
6 & 25 & 20 & \\
7 & 33 & 18 & 22 \\
8 & 29 & 23 & \\
\hline$\langle\Delta \beta / \beta\rangle$ & 24 & 16 & 20 \\
\hline
\end{tabular}

Table 2: Vertical beta-beating (in per cent) for the perturbed optics with $\beta_{y}^{*}=5 \mathrm{~cm}$ and KQSO.L2 $=-0.0004$ : multi-turn analysis, orthogonal kicks and results of the sextupole method (in each quadrant).

\section{RESONANT DISPERSION BUMPS}

During LEP start-up in 1992, large r.m.s. values of residual vertical dispersion (up to $60 \mathrm{~cm}$ ) have been observed, both with the $94 / 100$ optics and with the $91 / 97$ optics. It was later shown by simulation [5] that large fluctuations of $D_{y}$ can be generated when a reduced number of orbit correctors (typically 16) is used in each iteration. As a consequence, the initial strategy for closed orbit correction was modified (using 64 correctors per iteration) and the residual dispersion was much reduced. Meanwile we developed a resonant method of correction [6] that turned out to be very useful during physics runs.

Since $D_{y}$ is mainly driven by the vertical orbit harmon- 
ics closest to the vertical tune, we looked for special orbit bumps having a Fourier spectrum dominated by the line at the integer betatron tune, i.e., orbit bumps as close as possible to a pure betatron oscillation. The dispersion created by such a 'resonant' excitation could then be used to cancel the corresponding betatron component of the measured residual dispersion, by more than an order of magnitude, without any appreciable deterioration of the closed orbit (and of the coupling compensation). In order to apply this resonant excitation with the right phase, one has to determine the correct amplitude for two independent bumps in quadrature.

Let us consider a series of orbit bumps with the same amplitude, each of them extending over a large fraction of a machine arc. With the $90^{\circ}$ optics, each bump can be excited by two correctors, close to the beginning and to the end of the corresponding octant. For any given optics, and thus for given betatron phase advances across the straight sections, it is always possible to choose the relative phases of the arc bumps such that their contributions to dispersion add up almost coherently. In fact there are two independent choices giving rise to 'resonant families' of arc bumps in quadrature: the corresponding $D_{y}$ is either symmetric or antisymmetric around the IP's.

To estimate the amplification factor $A$, defined as the ratio between normalized dispersion (outside the bump) and normalized bump amplitude, we write the vertical closed orbit $y_{c o}(s)$ and the associated dispersion $D_{y}(s)$ for a single orbit bump with normalized amplitude $Y$, starting at the beginning $s_{i}$ of arc $i$ :

$$
\begin{aligned}
& y_{c o}(s)=Y \sqrt{\beta(s)} \sin \left[\mu(s)-\mu_{i}\right] \\
& D_{y}(s)=-y_{c o}(s)-\frac{Y \sqrt{\beta(s)}}{2 \sin (\pi Q)} \int_{b u m p} d s^{\prime}\left[\beta\left(K-K^{\prime} D_{x}\right)\right]_{s^{\prime}} f\left(s, s^{\prime}\right) \text {. }
\end{aligned}
$$

Here $f\left(s, s^{\prime}\right)=\cos \left[\pi Q-\left|\mu(s)-\mu\left(s^{\prime}\right)\right|\right] \sin \left[\mu\left(s^{\prime}\right)-\mu_{i}\right]=$ $=\left\{\sin \left[\pi Q+2 \mu\left(s^{\prime}\right)-\mu(s)-\mu_{i}\right]-\sin \left[\pi Q-\mu(s)+\mu_{i}\right]\right\} / 2$ (for $\mu(s)>\mu\left(s^{\prime}\right)$ ). The first term in curly brackets oscillates at twice the betatron frequency and thus changes sign at each cell (if the phase advance is $90^{\circ}$ ), while the second term is independent of the integration variable $s^{\prime}$. Therefore for a two-family sextupole arrangement, the contribution of the first term to the integral vanishes and neglecting the first term in the expression of $D_{y}$, we get

$$
\frac{D_{y}(s)}{\sqrt{\beta(s)}}=\frac{Y \sin \left[\pi Q-\mu(s)+\mu_{i}\right]}{4 \sin (\pi Q)} \int_{b u m p} d s^{\prime}\left[\beta\left(K-K^{\prime} D_{x}\right)\right]_{s^{\prime}}
$$

The last integral equals $4 \pi N_{\text {cell }} Q_{\text {cell }}^{\prime}$, where $N_{\text {cell }}$ is the (even) number of regular cells covered by the bump and $Q_{\text {ceil }}^{\prime}$ the chromaticity of a single cell. Thus the amplification factor $A$ for a vertical bump extending over a single arc is $A=\pi N_{\text {cell }} Q_{\text {ceil }}^{\prime} / \sin (\pi Q)$.

If the number of cells covered by orbit bumps in each octant is the same, the global amplification factor is $A_{\text {tot }}=$ $8 C A$, where the coherence factor $C \leq 1$ is given by

$$
C=\frac{1}{8} \sqrt{8+\sum_{i \neq j} p_{i} p_{j} \cos \left(\mu_{i}-\mu_{j}\right)}
$$

Here $p_{i}= \pm 1$ denotes the sign of the orbit bump starting at betatron phase $\mu_{i}$ in octant $i$. We choose the signs $p_{i}$ and the starting cells (i.e., the phases $\mu_{i}$ ) such that the coherence factor is as large as possible. For the $90^{\circ}$ injection optics and for an arc bump extending over 26 cells in each octant, our formula gives a global amplification factor $A_{\text {tot }} \sim 282$, while using MAD, we find global amplification factors of 280 and 276 , respectively, for symmetric and antisymmetric resonant bumps.

In the case of a four-family sextupole arrangement, as in the squeezed optics, the term oscillating at twice the betation frequency also contributes to vertical dispersion

$$
\begin{gathered}
\int_{b u m p} d s^{\prime}\left[\beta\left(K-K^{\prime} D_{x}\right)\right], \sin \left[\pi Q+2 \mu\left(s^{\prime}\right)-\mu(s)-\mu_{i}\right]= \\
-\frac{N_{\text {cell }}}{2} \int_{c e l l} d s^{\prime} \beta\left(s^{\prime}\right) \Delta K^{\prime}\left(s^{\prime}\right) D_{x}\left(s^{\prime}\right) \sin \left[\pi Q+2 \mu\left(s^{\prime}\right)-\mu(s)-\mu_{i}\right]
\end{gathered}
$$

where $\Delta K^{\prime}$ is the difference in sextupole strength between two SF or two SD families. This difference increases for decreasing values of $\beta^{*}$ and, if the bump phase $\mu_{i}$ is shifted by an odd number of cells (i.e., by a multiple of $\pi / 2$ ) the sign of $\Delta K^{\prime}$ is reversed. Since the main difference in sextupole strength occurs for the two SD families (corresponding to an effective three-family sextupole arrangement) and since the vertical phase advance from a vertical corrector to the defocussing sextupole in the same cell is almost $\pi / 2$, the contribution to vertical dispersion is proportional to the same trigonometric factor as the previous contribution due to $Q_{\text {cell }}^{\prime}$ and the amplification factor becomes

$$
A \simeq \frac{N_{c e l l}}{\sin (\pi Q)}\left|\pi Q_{c e l l}^{\prime} \pm \frac{1}{8}\left(\beta \Delta K^{\prime} L D_{x}\right)_{S D}\right|
$$

For the $5 \mathrm{~cm}$ squeezed optics, the cell chromaticity is $Q_{\text {cell }}^{\prime}=0.45$ and the contribution of the SD sextupoles is $\left(\beta \Delta K^{\prime} L D_{x}\right)_{S D} \simeq 6.5$. Therefore the symmetric and antisymumetric bumps now have rather different global amplification factors, approximately given by $A_{\text {tot }} \simeq 470 \pm 270$. These estimated factors are again very close to those computed by MAD, namely 203 and 735 .

\section{REFERENCES}

[1] D. Brandt, R. Giachino, E. Keil, G. Morpurgo, F. Ruggiero and A. Verdier,'Beta-beating in LEP for various optics configurations', SL-MD Note 61 (1992).

[2] A. Burns, 'BOM Software', in Proceedings of the Second Workshop on LEP Performance, Chamonix, 1992, Ed. J. Poole, SL/92-29 (DI), p. 95 (1992).

[3] H. Burkhardt and A. Verdier, SL-MD Note 31 (1992).

[4] E. Keil, 'Quadrupole position and beta-beating', SL-MD Note 83 (1993).

[5] E. Keil, SL-MD Note Il (1992).

[6] F. Ruggiero and A. Zholents, 'Resonant correction of residual dispersion in LEP', SL-MD Note 26 (1992). 